\title{
Appendix to: \\ The Economic Impacts of Climate Change: \\ Evidence from Agricultural Profits and Random Fluctuations in Weather*
}

Olivier Deschenes

University of California, Santa Barbara

Michael Greenstone

MIT and NBER

January 2007 


\section{Additional Tables}

\section{Appendix Table 1: Deviations of Growing Season Degree-Days and Precipitation From Normals}

\begin{tabular}{|c|c|c|c|c|c|c|c|c|}
\hline \multicolumn{9}{|c|}{ A. Removed Year Effects } \\
\hline \multirow[b]{2}{*}{ Degree Days } & \multicolumn{8}{|c|}{ Proportion of Counties with Degree Days Below/Above Average (Degrees): } \\
\hline & \pm 200 & \pm 400 & \pm 600 & \pm 800 & \pm 1000 & \pm 1200 & \pm 1400 & \pm 1600 \\
\hline 1987 & 0.477 & 0.231 & 0.122 & 0.069 & 0.045 & 0.024 & 0.015 & 0.006 \\
\hline 1992 & 0.531 & 0.302 & 0.203 & 0.123 & 0.049 & 0.023 & 0.008 & 0.004 \\
\hline 1997 & 0.426 & 0.173 & 0.084 & 0.046 & 0.027 & 0.012 & 0.008 & 0.003 \\
\hline \multirow[t]{2}{*}{2002} & 0.572 & 0.340 & 0.256 & 0.187 & 0.099 & 0.042 & 0.019 & 0.010 \\
\hline & \multicolumn{8}{|c|}{ Proportion of Counties with Precipitations Below/Above Average (Inches): } \\
\hline Total Precipitation & \pm 0.5 & \pm 1.0 & \pm 1.5 & \pm 2.0 & \pm 2.5 & \pm 3.0 & \pm 3.5 & \pm 4.0 \\
\hline 1987 & 0.856 & 0.715 & 0.572 & 0.455 & 0.345 & 0.255 & 0.189 & 0.148 \\
\hline 1992 & 0.828 & 0.653 & 0.506 & 0.391 & 0.310 & 0.239 & 0.178 & 0.121 \\
\hline 1997 & 0.849 & 0.697 & 0.552 & 0.420 & 0.314 & 0.230 & 0.163 & 0.119 \\
\hline 2002 & 0.845 & 0.702 & 0.561 & 0.450 & 0.351 & 0.281 & 0.217 & 0.161 \\
\hline \multicolumn{9}{|c|}{ B. Removed State*Year Effects } \\
\hline & \multicolumn{8}{|c|}{ Proportion of Counties with Degree Days Below/Above Average (Degrees): } \\
\hline Degree Days & \pm 200 & \pm 400 & \pm 600 & \pm 800 & \pm 1000 & \pm 1200 & \pm 1400 & \pm 1600 \\
\hline 1987 & 0.458 & 0.206 & 0.112 & 0.068 & 0.043 & 0.024 & 0.011 & 0.005 \\
\hline 1992 & 0.512 & 0.279 & 0.175 & 0.103 & 0.049 & 0.019 & 0.009 & 0.004 \\
\hline 1997 & 0.388 & 0.147 & 0.076 & 0.044 & 0.023 & 0.011 & 0.005 & 0.002 \\
\hline \multirow[t]{2}{*}{2002} & 0.559 & 0.348 & 0.239 & 0.156 & 0.082 & 0.034 & 0.016 & 0.008 \\
\hline & \multicolumn{8}{|c|}{ Proportion of Counties with Precipitations Below/Above Average (Inches): } \\
\hline Total Precipitation & \pm 0.5 & \pm 1.0 & \pm 1.5 & \pm 2.0 & \pm 2.5 & \pm 3.0 & \pm 3.5 & \pm 4.0 \\
\hline 1987 & 0.814 & 0.635 & 0.482 & 0.363 & 0.263 & 0.187 & 0.130 & 0.092 \\
\hline 1992 & 0.810 & 0.626 & 0.468 & 0.347 & 0.244 & 0.173 & 0.123 & 0.085 \\
\hline 1997 & 0.805 & 0.607 & 0.464 & 0.331 & 0.237 & 0.164 & 0.111 & 0.080 \\
\hline 2002 & 0.793 & 0.624 & 0.482 & 0.371 & 0.276 & 0.201 & 0.149 & 0.104 \\
\hline
\end{tabular}

Note: All statistics are weighted by acres of farmland. Panel A reports on the magnitude of the deviations between counties' yearly weather realizations and their long run averages after subtracting the deviation between the national average weather realization and the national long run average. The entries report the fraction of counties with deviations at least as large as the one reported in the column heading. For example, consider the 2002 degree-days row, it indicates that 57\%, 19\%, and 2\% of counties had deviations larger than 200, 800, and 1,400 degree days, respectively. Panel B repeats this exercise after subtracting the deviation between a states' yearly weather realization and the states' long run average (rather than the national deviation). 
Appendix Table 2: Sample Means by Quartiles of Temperature Normals

\begin{tabular}{|c|c|c|c|c|c|c|c|c|c|c|}
\hline \multirow[b]{2}{*}{ Quartile } & \multicolumn{5}{|c|}{$\underline{\text { January Temperature Normals }}$} & \multicolumn{5}{|c|}{ April Temperature Normals } \\
\hline & 1 & 2 & 3 & 4 & F-Stat & 1 & 2 & 3 & 4 & F-Stat \\
\hline \multicolumn{11}{|c|}{ Farmland values (\$1/ac): } \\
\hline Value of Land/Bldg & $1,196.9$ & $1,518.2$ & $1,403.3$ & $1,889.5$ & 20.7 & $1,040.5$ & $1,733.5$ & $1,802.0$ & $1,570.1$ & 52.6 \\
\hline \multicolumn{11}{|l|}{ Soil Characteristics: } \\
\hline K Factor & 0.30 & 0.33 & 0.33 & 0.28 & 39.3 & 0.30 & 0.32 & 0.33 & 0.29 & 23.9 \\
\hline Slope Length & 247.9 & 235.2 & 210.4 & 316.0 & 6.9 & 266.5 & 234.2 & 278.3 & 225.1 & 4.0 \\
\hline Fraction Flood-Prone & 0.10 & 0.14 & 0.19 & 0.19 & 14.3 & 0.11 & 0.13 & 0.20 & 0.18 & 12.4 \\
\hline Fraction Sand & 0.04 & 0.02 & 0.02 & 0.16 & 31.5 & 0.03 & 0.04 & 0.02 & 0.16 & 29.5 \\
\hline Fraction Clay & 0.21 & 0.16 & 0.20 & 0.36 & 28.5 & 0.19 & 0.19 & 0.22 & 0.34 & 15.4 \\
\hline Fraction Irrigated & 0.03 & 0.07 & 0.06 & 0.09 & 12.8 & 0.04 & 0.07 & 0.08 & 0.06 & 6.7 \\
\hline Permeability & 2.24 & 1.92 & 2.05 & 3.51 & 20.0 & 2.13 & 2.12 & 2.04 & 3.55 & 17.6 \\
\hline Moisture Capacity & 0.18 & 0.19 & 0.17 & 0.14 & 145.0 & 0.18 & 0.19 & 0.17 & 0.14 & 88.5 \\
\hline Salinity & 0.05 & 0.02 & 0.01 & 0.03 & 12.1 & 0.05 & 0.02 & 0.02 & 0.02 & 14.4 \\
\hline \multicolumn{11}{|c|}{ Socioeconomic and Locational Attributes: } \\
\hline Pop Density & 33.0 & 65.4 & 50.5 & 95.1 & 17.9 & 28.1 & 67.8 & 84.8 & 72.4 & 27.4 \\
\hline \multirow[t]{2}{*}{ Per Capita Income } & 16,573 & 16,755 & 15,136 & 15,280 & 15.4 & 16,432 & 16,648 & 16,193 & 14,525 & 20.8 \\
\hline & \multicolumn{5}{|c|}{ July Temperature Normals } & \multicolumn{5}{|c|}{ October Temperature Normals } \\
\hline Quartile & 1 & 2 & 3 & 4 & F-Stat & 1 & 2 & 3 & 4 & F-Stat \\
\hline \multicolumn{11}{|c|}{ Farmland values (\$1/ac): } \\
\hline Value of Land/Bldg & $1,120.0$ & $1,775.6$ & $1,606.9$ & $1,506.0$ & 26.0 & $1,018.5$ & $1,816.8$ & $1,653.9$ & $1,728.5$ & 60.3 \\
\hline \multicolumn{11}{|l|}{ Soil Characteristics: } \\
\hline K Factor & 0.32 & 0.30 & 0.32 & 0.30 & 13.8 & 0.31 & 0.31 & 0.33 & 0.29 & 20.2 \\
\hline Slope Length & 280.5 & 245.5 & 255.6 & 218.9 & 5.0 & 270.5 & 218.0 & 230.5 & 275.0 & 9.8 \\
\hline Fraction Sand & 0.03 & 0.05 & 0.03 & 0.14 & 23.9 & 0.03 & 0.04 & 0.03 & 0.15 & 24.1 \\
\hline Fraction Clay & 0.18 & 0.21 & 0.21 & 0.33 & 16.1 & 0.18 & 0.20 & 0.20 & 0.35 & 22.8 \\
\hline Fraction Irrigated & 0.05 & 0.06 & 0.07 & 0.06 & 3.4 & 0.04 & 0.06 & 0.06 & 0.08 & 6.0 \\
\hline Permeability & 2.05 & 2.34 & 2.04 & 3.28 & 15.8 & 2.14 & 2.09 & 2.11 & 3.43 & 15.2 \\
\hline Moisture Capacity & 0.17 & 0.18 & 0.18 & 0.15 & 63.6 & 0.18 & 0.19 & 0.17 & 0.14 & 81.4 \\
\hline Wetlands & 0.06 & 0.05 & 0.05 & 0.10 & 23.4 & 0.07 & 0.04 & 0.04 & 0.11 & 41.4 \\
\hline Salinity & 0.05 & 0.04 & 0.01 & 0.02 & 13.7 & 0.05 & 0.01 & 0.01 & 0.02 & 16.8 \\
\hline \multicolumn{11}{|c|}{ Socioeconomic and Locational Attributes: } \\
\hline Pop Density & 30.2 & 84.3 & 59.4 & 67.6 & 21.3 & 21.0 & 83.5 & 62.5 & 92.2 & 50.2 \\
\hline Per Capita Income & 16,583 & 16,483 & 16,118 & 14,741 & 17.8 & 16,319 & 16,813 & 15,989 & 14,857 & 12.7 \\
\hline
\end{tabular}

Notes: All dollar figures in 2002 constant dollars. The entries report the results of weighted regressions where the dependent variable is noted in the row headings and the weight is the square root of the acres of farmland. The entries are the parameter estimates from dummy variables for quartiles of the relevant climate normal, so they report the mean of each variable by quartile. Climate normals are defined as the 1970-2000 average of temperature and precipitation, by county. The F-statistics are from tests of equality of the means across the quartiles. The regressions are fit with data from the 1978, 1982, 1987, 1992, 1997, and 2002 Censuses, so they adjust for year fixed effects to account for national differences across years. The variance-covariance matrix allows for a county-specific variance component. See the text for further details. 
Appendix Table 3: Sample Means by Quartiles of Precipitation Normals

\begin{tabular}{|c|c|c|c|c|c|c|c|c|c|c|}
\hline \multirow[b]{2}{*}{ Quartile } & \multicolumn{5}{|c|}{$\underline{\text { January Precipitation Normals }}$} & \multicolumn{5}{|c|}{ April Precipitation Normals } \\
\hline & 1 & 2 & 3 & 4 & F-Stat & 1 & 2 & 3 & 4 & F-Stat \\
\hline \multicolumn{11}{|c|}{ Farmland values (\$1/ac): } \\
\hline Value of Land/Bldg & 914.3 & $1,708.4$ & 2,366.4 & 2,228.5 & 217.2 & $1,026.7$ & $1,717.3$ & 2,335.8 & $1,962.7$ & 148.9 \\
\hline \multicolumn{11}{|l|}{$\underline{\text { Soil Characteristics: }}$} \\
\hline K Factor & 0.30 & 0.32 & 0.32 & 0.29 & 15.0 & 0.31 & 0.29 & 0.31 & 0.35 & 90.7 \\
\hline Slope Length & 261.3 & 258.4 & 244.9 & 209.6 & 3.2 & 312.0 & 195.9 & 189.4 & 164.7 & 46.1 \\
\hline Fraction Flood-Prone & 0.12 & 0.15 & 0.19 & 0.22 & 12.6 & 0.13 & 0.12 & 0.13 & 0.27 & 35.1 \\
\hline Fraction Sand & 0.03 & 0.06 & 0.06 & 0.18 & 29.3 & 0.05 & 0.11 & 0.07 & 0.02 & 42.5 \\
\hline Fraction Clay & 0.26 & 0.21 & 0.18 & 0.19 & 6.3 & 0.23 & 0.27 & 0.20 & 0.16 & 11.8 \\
\hline Fraction Irrigated & 0.05 & 0.05 & 0.08 & 0.08 & 4.5 & 0.08 & 0.05 & 0.02 & 0.05 & 40.1 \\
\hline Permeability & 2.13 & 2.28 & 2.59 & 3.65 & 18.9 & 2.32 & 2.99 & 2.52 & 1.75 & 28.3 \\
\hline Moisture Capacity & 0.18 & 0.18 & 0.16 & 0.15 & 50.3 & 0.17 & 0.17 & 0.18 & 0.18 & 19.8 \\
\hline Wetlands & 0.04 & 0.06 & 0.08 & 0.17 & 73.2 & 0.04 & 0.09 & 0.09 & 0.10 & 49.8 \\
\hline Salinity & 0.04 & 0.02 & 0.01 & 0.01 & 25.7 & 0.05 & 0.01 & 0.00 & 0.00 & 45.7 \\
\hline \multicolumn{11}{|c|}{ Socioeconomic and Locational Attributes: } \\
\hline Pop Density & 13.4 & 80.8 & 139.2 & 101.2 & 94.9 & 24.9 & 72.3 & 138.2 & 85.5 & 62.1 \\
\hline \multirow[t]{2}{*}{ Per Capita Income } & 16,087 & 16,236 & 16,364 & 14,976 & 8.0 & 15,963 & 16,670 & 16,940 & 14,340 & 64.9 \\
\hline & \multicolumn{5}{|c|}{$\underline{\text { July Precipitation Normals }}$} & \multicolumn{5}{|c|}{ October Precipitation Normals } \\
\hline Quartile & 1 & 2 & 3 & 4 & F-Stat & 1 & 2 & 3 & 4 & F-Stat \\
\hline \multicolumn{11}{|c|}{ Farmland values (\$1/ac): } \\
\hline Value of Land/Bldg & $1,115.4$ & $1,402.6$ & $2,233.6$ & $2,166.2$ & 139.5 & $1,030.7$ & $1,994.9$ & $1,957.2$ & $1,926.8$ & 110.7 \\
\hline \multicolumn{11}{|l|}{ Soil Characteristics: } \\
\hline K Factor & 0.32 & 0.30 & 0.32 & 0.25 & 38.6 & 0.31 & 0.29 & 0.30 & 0.33 & 25.2 \\
\hline Slope Length & 325.6 & 194.2 & 185.6 & 161.4 & 53.9 & 309.6 & 210.5 & 181.3 & 169.1 & 44.3 \\
\hline Fraction Flood-Prone & 0.16 & 0.14 & 0.16 & 0.12 & 3.9 & 0.14 & 0.08 & 0.16 & 0.24 & 52.5 \\
\hline Fraction Sand & 0.03 & 0.04 & 0.03 & 0.28 & 45.3 & 0.03 & 0.11 & 0.11 & 0.04 & 26.3 \\
\hline Fraction Clay & 0.29 & 0.17 & 0.21 & 0.10 & 35.2 & 0.22 & 0.25 & 0.15 & 0.29 & 14.6 \\
\hline Fraction Irrigated & 0.07 & 0.06 & 0.02 & 0.04 & 18.7 & 0.08 & 0.02 & 0.02 & 0.05 & 29.0 \\
\hline Permeability & 1.93 & 2.36 & 1.89 & 5.23 & 49.6 & 2.14 & 2.89 & 3.03 & 2.15 & 12.6 \\
\hline Moisture Capacity & 0.16 & 0.19 & 0.19 & 0.15 & 122.7 & 0.17 & 0.18 & 0.16 & 0.16 & 12.6 \\
\hline Wetlands & 0.03 & 0.07 & 0.10 & 0.17 & 143.7 & 0.04 & 0.09 & 0.10 & 0.11 & 80.5 \\
\hline Salinity & 0.05 & 0.02 & 0.00 & 0.00 & 46.7 & 0.05 & 0.01 & 0.01 & 0.01 & 35.1 \\
\hline \multicolumn{11}{|c|}{ Socioeconomic and Locational Attributes: } \\
\hline Pop Density & 33.6 & 51.0 & 117.0 & 104.8 & 47.3 & 21.2 & 97.0 & 99.9 & 102.6 & 67.8 \\
\hline Per Capita Income & 15,835 & 16,391 & 16,665 & 15,260 & 11.0 & 16,299 & 16,300 & 15,415 & 15,370 & 8.1 \\
\hline
\end{tabular}

Notes: All dollar figures in 2002 constant dollars. The entries report the results of weighted regressions where the dependent variable is noted in the row headings and the weight is the square root of the acres of farmland. The entries are the parameter estimates from dummy variables for quartiles of the relevant climate normal, so they report the mean of each variable by quartile. Climate normals are defined as the 1970-2000 average of temperature and precipitation, by county. The F-statistics are from tests of equality of the means across the quartiles. The regressions are fit with data from the 1978, 1982, 1987, 1992, 1997, and 2002 Censuses, so they adjust for year fixed effects to account for national differences across years. The variance-covariance matrix allows for a county-specific variance component. See the text for further details. 
Appendix Table 4: Fixed-Effects Estimates of Agricultural Profit Models and Hypothesis Tests

\begin{tabular}{|c|c|c|c|c|}
\hline & $(1)$ & $(2)$ & (3) & $(4)$ \\
\hline \multicolumn{5}{|l|}{ Non-irrigated counties: } \\
\hline$\overline{\text { Growing Season Degree-Days }}$ & $\begin{array}{c}-0.00295560 \\
(0.00215640)\end{array}$ & $\begin{array}{c}-0.00293180 \\
(0.00216900)\end{array}$ & $\begin{array}{c}0.00236230 \\
(0.00201700)\end{array}$ & $\begin{array}{c}0.00292280 \\
(0.00200150)\end{array}$ \\
\hline Growing Season Degree-Days Squared & $\begin{array}{c}0.00000020 \\
(0.00000033)\end{array}$ & $\begin{array}{c}0.00000018 \\
(0.00000033)\end{array}$ & $\begin{array}{l}-0.00000015 \\
(0.00000031)\end{array}$ & $\begin{array}{c}-0.00000023 \\
(0.00000031)\end{array}$ \\
\hline Growing Season Total Precipitation & $\begin{array}{c}-0.46070710 \\
(0.41975300)\end{array}$ & $\begin{array}{c}-0.45038850 \\
(0.42017730)\end{array}$ & $\begin{array}{c}0.01624210 \\
(0.46391920)\end{array}$ & $\begin{array}{c}0.03287530 \\
(0.47133400)\end{array}$ \\
\hline Growing Season Total Precipitation Squared & $\begin{array}{c}0.00243360 \\
(0.00975000)\end{array}$ & $\begin{array}{c}0.00230540 \\
(0.00973340)\end{array}$ & $\begin{array}{c}0.00360220 \\
(0.01084080)\end{array}$ & $\begin{array}{c}0.00331670 \\
(0.01095420)\end{array}$ \\
\hline \multicolumn{5}{|l|}{ Irrigated counties: } \\
\hline Growing Season Degree-Days & $\begin{array}{c}-0.01159260 \\
(0.00877660)\end{array}$ & $\begin{array}{c}-0.01169860 \\
(0.00879800)\end{array}$ & $\begin{array}{c}-0.00366090 \\
(0.00801680)\end{array}$ & $\begin{array}{l}-0.00368220 \\
(0.00800660)\end{array}$ \\
\hline Growing Season Degree-Days Squared & $\begin{array}{c}0.00000123 \\
(0.00000141)\end{array}$ & $\begin{array}{c}0.00000125 \\
(0.00000142)\end{array}$ & $\begin{array}{c}0.00000020 \\
(0.00000129)\end{array}$ & $\begin{array}{c}0.00000019 \\
(0.00000129)\end{array}$ \\
\hline Growing Season Total Precipitation & $\begin{array}{c}0.63382370 \\
(1.29355200)\end{array}$ & $\begin{array}{c}0.77529660 \\
(1.30510900)\end{array}$ & $\begin{array}{c}1.75161400 \\
(1.31468900)\end{array}$ & $\begin{array}{c}1.84390400 \\
(1.32800800)\end{array}$ \\
\hline Growing Season Total Precipitation Squared & $\begin{array}{c}0.00074260 \\
(0.03620790)\end{array}$ & $\begin{array}{c}-0.00378570 \\
(0.03658910)\end{array}$ & $\begin{array}{l}-0.01862300 \\
(0.03677150)\end{array}$ & $\begin{array}{c}-0.02049190 \\
(0.03718890)\end{array}$ \\
\hline \multicolumn{5}{|c|}{$\underline{\text { P-Values from Tests of Equality of Marginal Effects by Irrigation Status: }}$} \\
\hline Degree Days & 0.165 & 0.173 & 0.054 & 0.047 \\
\hline Total Precip & 0.012 & 0.012 & 0.009 & 0.007 \\
\hline \multicolumn{5}{|c|}{ P-Values from Tests that Listed Variables are Jointly Equal to Zero: } \\
\hline Soil Characteristics: & --- & 0.162 & --- & 0.542 \\
\hline County Fixed Effects & 0.001 & 0.001 & 0.001 & 0.001 \\
\hline Year Fixed Effects & 0.001 & 0.001 & --- & --- \\
\hline State by Year Fixed Effects & --- & --- & 0.001 & 0.001 \\
\hline Soil Controls & No & Yes & No & Yes \\
\hline County Fixed Effects & Yes & Yes & Yes & Yes \\
\hline Year Fixed Effects & Yes & Yes & No & No \\
\hline State*Year Fixed Effects & No & No & Yes & Yes \\
\hline
\end{tabular}

Notes: All dollar figures in 2002 constant dollars. The entries in the first panel are the result of the estimation of version of equation (4) for agricultural profits that model the growing season degree-days and total precipitation with quadratics. The second and third panels report p-values from F-tests that subsets of the variables are jointly equal to zero. The controls are listed in the row headings at the bottom of the table. See the text for further details. 